\title{
PILHAS ALCALINAS: UM DISPOSITIVO ÚTIL PARA O ENSINO DE QUÍMICA
}

\author{
Elaine Y. Matsubara, Cláudio R. Neri e J. Maurício Rosolen* \\ Departamento de Química, Faculdade de Filosofia Ciências e Letras de Ribeirão Preto, Universidade de São Paulo,
} Av. Bandeirantes, 3900, 14040-901 Ribeirão Preto - SP, Brasil

Recebido em 26/4/06; aceito em 15/9/06; publicado na web em 26/3/07

\begin{abstract}
ALKALINE BATTERY: A USEFUL DEVICE IN THE TEACHING OF CHEMISTRY. This paper presents the alkaline battery $\left(\mathrm{MnO}_{2} / \mathrm{Zn}\right)$ as a useful device in the teaching of chemistry. The preparation of the battery, the materials used in the preparation of the $\mathrm{MnO}_{2}$ electrode, the mechanism of energy storage and the parameters often used in the understanding of general batteries are discussed in detail. In addition, a schedule and a questionnaire that can be applied in an experimental class have been developed, which allow the assembly of an alkaline battery, its discharge using a galvanostatic or a load-resistance procedure, and the elaboration of a report based on the main text. This experimental class has been offered in the chemistry course of FFCLRP.
\end{abstract}

Keywords: alkaline battery; $\mathrm{MnO}_{2}$; graphite

\section{INTRODUÇÃO}

A maioria dos programas dos cursos de graduação em Química contém pouca informação sobre a conversão de energia química em elétrica e sobre a importância das propriedades físico-químicas dos materiais que compõem estes dispositivos. Nos vários textos didáticos, as baterias e pilhas são utilizadas principalmente para a discussão de reações redox e dos aspectos cinéticos e termodinâmicos associados à passagem de corrente elétrica em circuitos externos $^{1-3}$. Os dispositivos normalmente usados para tal fim são: a bateria de chumbo-ácido $\mathrm{E}_{\text {cela }}=1,98 \mathrm{~V}\left(\mathrm{~Pb}_{\text {(sólido) }} \mid \mathrm{PbSO}_{4}\right.$ $\left.\mathrm{H}_{2} \mathrm{SO}_{4(\text { aquoso) }}\left|\mathrm{PbSO}_{4 \text { (sólido) }}\right| \mathrm{PbO}_{2 \text { (sólido) }} \mid \mathrm{Pb}_{\text {(sólido) }}\right)$, pilha de Daniel $\mathrm{E}_{\text {cela la }}=$

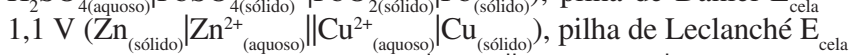
na faixa de 1,55-1,74 V ( $\left.\mathrm{Zn}_{\text {(sólido) }}\left|\mathrm{ZnCl}_{2}\right|\left|\mathrm{NH}_{4} \mathrm{Cl}_{\text {(aquoso) }}\right| \mathrm{MnO}_{2}, \mathrm{C}_{\text {(sólido) }}\right)$, onde $\mathrm{E}_{\text {cela }}$ é o potencial de circuito aberto ${ }^{4}$. Contudo, conceitos como capacidade de carga/descarga, densidades de energia e potência são pouco discutidos ou então nem mencionados, assim como ocorre com os fatores que provocam desvios na termodinâmica das celas eletroquímicas e com as características estruturais e morfológicas dos materiais usados na preparação dos dispositivos de conversão de energia. Neste contexto é que o presente trabalho foi realizado, com o objetivo de fornecer apoio didático para a abordagem e aprimoramento de conceitos importantes na formação de um químico: a armazenagem de energia e as reações de oxidação/redução associadas à inserção de espécies iônicas em materiais, as implicações que o processamento e a síntese de um dado composto e eletrodo podem apresentar no desempenho de uma bateria, o uso da Lei de Faraday na determinação de parâmetros importantes para a descrição de baterias, o efeito Joule e a energia livre de Gibbs. Enfim, o trabalho insere o aluno em uma área de grande interesse tecnológico, fornecendo suporte para a preparação de pilhas alcalinas utilizando um protocolo muito próximo daquele usado pela indústria.

\section{PILHAS ALCALINAS}

A pilha alcalina nada mais é do que uma variação da pilha de Leclanchè de $1860^{4,5}$ porque ambas utilizam como materiais eletródicos ativos $\mathrm{Zn}$ e $\mathrm{MnO}_{2}$. A pilha de Leclanchè e suas varia-

*e-mail: rosolen@ffclrp.usp.br ções geram corrente elétrica devido à oxidação do $\mathrm{Zn} \rightarrow \mathrm{Zn}^{2+}+2 \mathrm{e}$ e a redução do $\mathrm{Mn}^{4+}+2 \mathrm{e} \rightarrow \mathrm{Mn}^{2+}$. Entretanto, cada tipo de pilha $\mathrm{MnO}_{2} / \mathrm{Zn}$ gera produtos durante sua descarga, que dependem do eletrólito utilizado. Para uma pilha cujo eletrólito é uma solução concentrada de $\mathrm{KOH}$ (alcalina), a reação de cela tem sido representada por: $\mathrm{Zn}+2 \mathrm{MnO}_{2}+\mathrm{H}_{2} \mathrm{O} \rightarrow \mathrm{ZnO}+2 \mathrm{MnO}$. $(\mathrm{OH})$, em uma faixa de descarga de 1,5-0,9 V; ou seja, durante a descarga ocorre formação de $\mathrm{ZnO}\left(\mathrm{Zn}^{2+}+2 \mathrm{OH}^{-} \rightarrow \mathrm{ZnO}+\mathrm{H}_{2} \mathrm{O}\right)$ e a redução do $\mathrm{Mn}^{4+}$ a $\mathrm{Mn}^{3+}$ devido a uma reação de estado sólido associada com a inserção de prótons no dióxido de manganês, isto é, $\mathrm{H}_{\mathrm{x}} \mathrm{MnO}_{2}$. Por outro lado, estudos recentes propuseram que o mecanismo de descarga de pilha alcalina envolve, na verdade, a inserção de Zn no cátodo de $\mathrm{MnO}_{2}$. Todavia, quando a descarga envolver uma transferência de elétrons acima de $0,5 \mathrm{~mol}$, a reação é de troca iônica entre cátions, que leva à formação do composto $\mathrm{ZnMn}_{2} \mathrm{O}_{4}^{7}$. Deste modo, a descarga da pilha alcalina apresenta um tipo de troca catiônica pouco comum, mas que pode ser encontrada também em eletrodos de hidróxido de níquel em meio alcalino ${ }^{8}$. O próton já intercalado no interior do $\mathrm{MnO}_{2}$ migra para o eletrólito, enquanto íons de $\mathrm{Zn}^{2+}$ do eletrólito são inseridos no $\mathrm{MnO}_{2}$, um fenômeno que ocorre durante a polarização do eletrodo. Para uma descarga envolvendo mais que 1e', o mecanismo de reação no cátodo envolve formação de $\mathrm{Mn}(\mathrm{OH})_{2}$. Se a descarga tiver como limite inferior de voltagem valores na faixa de $1,1-1,0 \mathrm{~V}$, esta reação de inserção é reversível e a pilha alcalina poderá funcionar como uma bateria que pode ser carregada, ou seja, uma bateria secundária ${ }^{6}$.

Tecnologicamente, a pilha alcalina merece destaque porque apresenta baixo impacto ambiental frente a outros tipos de baterias primárias (baterias não recarregáveis), boa capacidade de descarga (224 Ah $\mathrm{kg}^{-1}$ ), pequena perda de carga durante o armazenamento e pode ser produzida em vários tamanhos e formas (cilíndrica, botões). Estes fatores explicam seu grande sucesso comercial. A Figura 1 ilustra os principais componentes de uma pilha alcalina do tipo comercial.

Nas celas comerciais o cátodo é composto de uma mistura de $\mathrm{MnO}_{2}$ de alta pureza, de carbono condutor e aditivos, e tem a forma de um cilindro que é previamente moldado sobre pressão. $\mathrm{O}$ ânodo é predominantemente constituído por um gel à base de pó de $\mathrm{Zn}$, solução 9,0 $\mathrm{mol} \mathrm{L}^{-1}$ de $\mathrm{KOH}$ e carboxi-metil celulose de sódio-CMC que confere à mistura a textura de pasta ou cimento, possibilitando a imobilização do eletrólito. 


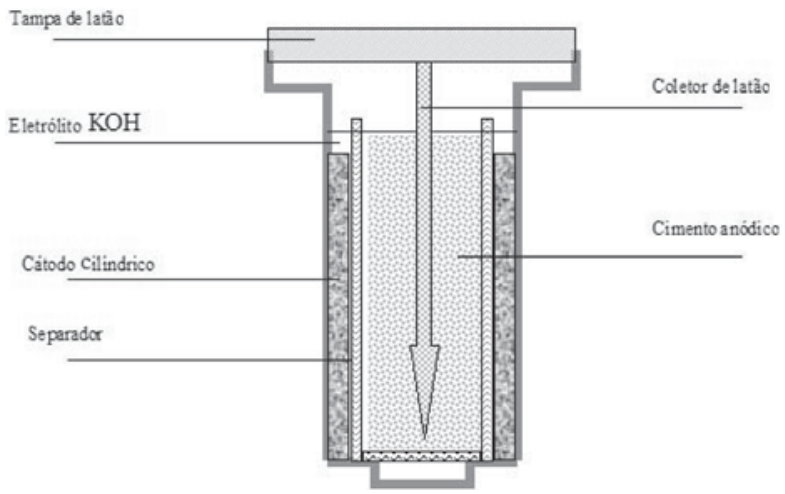

Figura 1. Ilustração de uma pilha alcalina comercial de $\mathrm{MnO} / \mathrm{Zn}$. O cátodo é um cilindro vazado no qual vem inserido um casulo (separador) que contém o cimento de $\mathrm{Zn}$. O coletor de corrente do cátodo é feito de aço revestido de Ni ou de uma tinta especial, à base de grafite. A pilha contém um reservatório para eletrólito na parte superior

Já as baterias alcalinas secundárias de $\mathrm{MnO}_{2} / \mathrm{Zn}$ utilizam no cátodo: $\mathrm{MnO}_{2}$, grafite, $\mathrm{BaSO}_{4}$ e $\mathrm{Ag}_{2} \mathrm{O}$; no ânodo: pó de $\mathrm{Zn}$, polietilenoglicol, solução de $\operatorname{In}_{2}\left(\mathrm{SO}_{4}\right)_{3}, \mathrm{KOH} 9,0$ mol L ${ }^{-1}$ com $5 \%$ de $\mathrm{ZnO}$ e agentes quelantes como trietilamina. Em ambas as baterias existem separadores entre os eletrodos, que atuam como um casulo, envolvendo o ânodo.

Nas pilhas alcalinas e na maioria das baterias, as propriedades físicas e químicas dos materiais utilizados são muito importantes. Nos eletrodos, por ex., estas propriedades são: a pureza, a morfologia (tamanho e forma dos grãos), a composição química e o tipo de estrutura dos materiais, sendo que o dióxido de manganês $\left(\mathrm{MnO}_{2}\right)$ e o carbono, que é usado para aumentar a condutividade eletrônica do cátodo, merecem uma discussão especial.

$\mathrm{O} \mathrm{MnO}_{2}$ é um óxido isolante que pode apresentar vários tipos de estrutura cristalina: alfa, beta e gama. $\mathrm{O} \alpha-\mathrm{MnO}_{2}$ (simetria tetragonal, grupo espacial $\mathrm{I} 4 / \mathrm{m}$ ) é uma variante que apresenta estrutura onde duplas cadeias do octaedro $\mathrm{MnO}_{6}$ estão interligadas, formando canais unidimensionais que se estendem paralelamente ao eixo cristalográfico e na cela unitária. Esta fase pode ser encontrada na forma mineral como $\mathrm{BaMn}_{8} \mathrm{O}_{16}, \mathrm{KMn}_{8} \mathrm{O}_{16}$, por reação de $\mathrm{Mn}_{2} \mathrm{O}_{3}$ ou $\mathrm{Li}_{2} \mathrm{MnO}_{3} \mathrm{em}$ soluções concentradas de ácido sulfúrico. Tanto na forma mineral como sintética do $\alpha-\mathrm{MnO}_{2}$ verifica-se a presença de canais de intercalação no interior da estrutura, que podem estar eventualmente preenchidos com íons $\mathrm{Ba}, \mathrm{K}$ ou moléculas de $\mathrm{H}_{2} \mathrm{O}$. Estes íons prejudicam a inserção de cátions de pequena dimensão, como prótons ou lítio, e a estrutura mostra-se relativamente pouco estável frente à reação de intercalação das espécies iônicas. Isto se explica porque a fase $\alpha$ do $\mathrm{MnO}_{2}$ não é utilizada em pilhas alcalinas ou baterias de Li comerciais9. Uma outra fase do $\mathrm{MnO}_{2}$, que também não encontra aplicação econômica nas pilhas e baterias, é a $\beta-\mathrm{MnO}_{2}$ que tem estrutura tipo rutilo com simetria tetragonal ${ }^{10}$. Já a fase $\gamma-$ $\mathrm{MnO}_{2}$ apresenta uma estrutura que é uma mistura de fase rutilo$\mathrm{MnO}_{2}$ e ramsdelite ${ }^{11}$, que apresenta canais unidimensionais adequados para inserção de prótons ou lítio. Assim, é esta fase a utilizada na produção de eletrodos de pilhas alcalinas comerciais. A sua obtenção é atualmente realizada em escala, empregando-se eletrodeposição em reator de fluxo e seu nome comercial é bióxido de manganês eletrolítico ou BME (ou EMD, em inglês ${ }^{12}$ ).

Já o carbono condutor utilizado nas pilhas tem grandes variações, mas todos possuem elevada condutividade eletrônica. Nas pilhas alcalinas atuais utiliza-se predominantemente a grafite natural ou sintética. A forma e a distribuição de tamanho dos grãos dos materiais usados na preparação do cátodo e ânodo são parâmetros que dependem do fabricante e não são divulgados. A grafite da pilha alcalina tem a finalidade de reduzir ao máximo a resistência elétrica da pastilha de $\mathrm{MnO}_{2}$, sem significativa redução da percolação do eletrólito de $\mathrm{KOH}$ no interior do eletrodo, uma vez que a superfície da grafite é hidrofóbica. Assim, as propriedades morfológicas, estruturais e o grau de pureza de uma grafite têm grande influência na durabilidade e na resposta elétrica de uma pilha alcalina, como será discutido. A Tabela 1 apresenta exemplos de grafite de origem mineral cuja diferenciação é definida por parâmetros de interesse na preparação do eletrodo $\mathrm{MnO}_{2}$ /grafite.

Tabela 1. Exemplos de grafites usadas em pilhas alcalinas

\begin{tabular}{lrcccrrc}
\hline Grafite & \multicolumn{1}{c}{$\begin{array}{c}\text { BET } \\
\left(\mathrm{m}^{2} \mathrm{~g}^{-1}\right)\end{array}$} & $\% \mathrm{C}$ & $\begin{array}{c}\mathrm{d}_{002} \\
(\AA)\end{array}$ & $\begin{array}{c}\mathrm{d}_{10} \\
(\mu \mathrm{m})\end{array}$ & $\begin{array}{c}\mathrm{d}_{50} \\
(\mu \mathrm{m})\end{array}$ & $\begin{array}{c}\mathrm{d}_{90} \\
(\mu \mathrm{m})\end{array}$ & $\begin{array}{c}\mathrm{L}_{\mathrm{c}} \\
(\AA)\end{array}$ \\
\hline A & 16,9 & 100 & 3,3580 & 1,74 & 3,81 & 7,15 & 1244 \\
B & 4,8 & 100 & 3,3560 & 8,23 & 20,44 & 44,67 & 2896 \\
C & 4,1 & 88,1 & 3,3620 & 8,27 & 21,05 & 46,01 & 4251 \\
D & 9,8 & 94,9 & 3,3628 & 3,53 & 7,20 & 12,85 & 2213 \\
E & 10,5 & 81,7 & 3,3576 & 3,30 & 6,75 & 12,18 & 2019
\end{tabular}

O parâmetro $\mathrm{d}_{002}$ é a distância interplanar na grafite. Os parâmetros

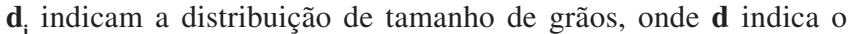
diâmetro médio das partículas no pó e i corresponde à percentagem das partículas com diâmetro abaixo de $\mathbf{d}_{\mathrm{i}}$. Lc é definido como comprimento de coerência dos cristalitos ou a altura do empilhamento de $\mathrm{m}$ camadas de grafite e relaciona-se $\mathrm{a} \mathrm{d}_{002}$ (distância interplanar da grafite) pela equação $\mathrm{Lc}=\mathrm{d}_{002}(\mathrm{~m}-1)$, onde $\mathrm{m}$ é número de camadas de grafeno. Os parâmetros $L c$ e $\mathrm{d}_{002}$ são determinados pela Equação de Scherrer corrigida utilizando-se um padrão de pó de Si e a Equação de Bragg utilizando-se comprimento de onda $1,548 \AA^{13}$. BET é a área superficial específica.

A Figura 2 mostra a forma dos cristais de grafite que podem ser usados na preparação do eletrodo $\mathrm{MnO}_{2}$ da pilha alcalina. Notase que os grãos da grafite são produzidos na forma de pequenos discos, fato que facilita a preparação do eletrodo (pastilhamento, compactação, mistura com o $\mathrm{MnO}_{2}$ ).

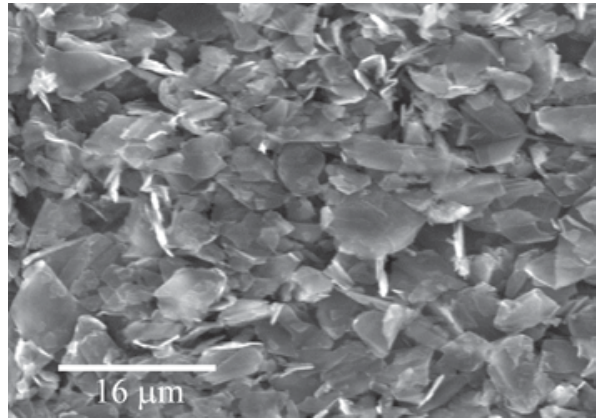

Figura 2. Fotografia de microscopia de varredura eletrônica da grafite $D$ (Tabela 1)

\section{PARÂMETROS DE INTERESSE PARA A AVALIAÇÃO DO DESEMPENHO DE BATERIAS}

No estudo de baterias existe uma série de parâmetros que definem o desempenho prático de uma bateria ou do seu eletrodo, unidades que são úteis também para o entendimento de alguns conceitos termodinâmicos.

A capacidade nominal (mAh) de uma bateria ou eletrodo é definida como a quantidade de corrente que pode ser fornecida em um dado intervalo de tempo. Este parâmetro é determinado experimentalmente, medindo-se o tempo de descarga da bateria em um 
certo valor de corrente (o produto destes fornece a capacidade nominal). Por ex., se uma bateria consegue fornecer uma descarga de 0,5 A por $30 \mathrm{~min}$, a capacidade nominal desta será $500 \mathrm{~mA} \times 0,5 \mathrm{~h}$, ou seja, $250 \mathrm{mAh}$. Por outro lado, esta mesma bateria é também capaz de fornecer $250 \mathrm{~mA}$ por $1 \mathrm{~h}$, todavia, apresentando uma variação de voltagem menor que a observada no caso de $0,5 \mathrm{~A}$.

Um outro parâmetro também utilizado, só que para avaliação energética específica dos eletrodos, é a capacidade específica. Esta capacidade é a capacidade nominal do eletrodo pela massa de material ativo $\left(\mathrm{MnO}_{2}\right)$, expressa em $\mathrm{mAh} \mathrm{g}^{-1}$, e pode ser obtida durante a descarga/carga do eletrodo, geralmente em condições onde a corrente é mantida constante. Pode também pode ser calculada utilizandose a Lei de Faraday aplicada à reação de transferência de carga que ocorre no eletrodo. Assim, para as reações de oxidação/redução associadas à conversão de energia, a capacidade específica é igual a ne x F / (3600 x MM) onde, $\mathrm{MM}=$ massa molar $\left(\mathrm{g} \mathrm{mol}^{-1}\right), \mathrm{F}=96485 \mathrm{C}$ (Coulombs) e ne $=$ número de elétrons trocados na reação de inserção de prótons no $\mathrm{MnO}_{2}$. Com a capacidade nominal da pilha e a capacidade específica teórica é possível obter, por ex., a massa de $\mathrm{MnO}_{2}$ usada na preparação de uma pilha comercial.

Além destes parâmetros, utiliza-se no estudo de pilhas e baterias a densidade de potência ou potência específica, em $\mathrm{W} \mathrm{kg}^{-1}$, que pode ser determinada multiplicando-se a corrente de descarga da bateria pela sua diferença de potencial médio, em Volts, durante a descarga. Já a densidade de energia gravimétrica, por outro lado, é expressa em $\mathrm{Wh} \mathrm{kg}^{-1} \mathrm{e}$ é determinada por alguns autores a partir da diferença de potencial entre cátodo e ânodo, pela corrente e o tempo (produto destes parâmetros). Outros preferem determiná-la multiplicando-se a capacidade específica pela voltagem média durante a descarga. Enfim, um outro parâmetro importante é a taxa de descarga (ou de carga), ou seja, a capacidade nominal dividida pelo tempo de descarga. Se uma pilha for descarregada usando uma taxa de $\mathrm{C} / 10$, isto significa que a mesma é capaz de fornecer a capacidade nominal $\mathrm{C}$ em $10 \mathrm{~h}$. Ainda, utilizando a capacidade nominal, (ex: $\mathrm{C}=750 \mathrm{mAh}$ ) podemos obter a corrente de descarga $\mathrm{I}_{\text {descarga }}=750 \mathrm{mAh} / 10 \mathrm{~h}=75 \mathrm{~mA}$.

Com estas grandezas é possível compreender como é realizada a escolha de uma bateria para uma dada aplicação. Por ex., muitos dos componentes e assessórios usados em veículos podem ter funcionamento impróprio se a voltagem da bateria estiver abaixo de $10 \mathrm{~V}$. Assim, com base na variação máxima de voltagem em conjunto com as correntes envolvidas no acionamento do motor e a operação dos demais componentes é que se escolhe o tipo de bateria. Isto explica porque normalmente motores de $2,0 \mathrm{~L}$ usam baterias de chumbo ácido de $56 \mathrm{Ah}$, enquanto motores de 1,0 L funcionam sem problemas com baterias de capacidade nominal menor. Quanto maior a capacidade nominal maior será o valor de corrente que uma bateria pode fornecer em um dado intervalo.

É interessante recordar que alguns destes parâmetros têm correlação com a energia livre de Gibbs. A função de Gibbs relaciona a força eletromotriz de uma bateria em descarga pela equação $\mathrm{dG}=-$ EFdn, no qual F é a constante de Faraday, E o potencial da cela em corrente zero e dn a variação da concentração em número de mols de carga (elétrons) que flui sob o potencial elétrico de forma reversível. Para um processo reversível, a variação de energia livre de Gibbs está associada ao trabalho não associado com pressão e volume que o sistema pode realizar. Isto significa que o trabalho máximo que pode ser extraído de uma bateria em descarga ocorre quando não existe outro processo irreversível paralelo à conversão de energia química em elétrica, como por ex., a geração de calor ou efeito Joule $\left(\mathrm{iR}^{2}\right)$. Quando a capacidade específica de um eletrodo é idêntica à obtida experimentalmente, não necessariamente podemos dizer do ponto de vista termodinâmico que se trata de um processo reversível. Isto ocorre porque o trabalho previsto pela energia livre de Gibbs não corresponderá ao valor que pode ser extraído efetivamente da bateria. Sempre existe uma queda-ôhmica ou uma resistência que acaba consumindo a energia que pode ser usada na forma de trabalho. Tal fato explica porque é tão importante a preparação de baterias com a menor resistência possível. Finalmente, quanto maior for a potência específica ou a densidade específica, maior será a capacidade de realizar trabalho por parte da bateria.

\section{CAPACIDADE ESPECÍFICA NA PILHA ALCALINA}

Para as pilhas alcalinas nas condições estudadas, o número de elétrons envolvidos nos eletrodos de $\mathrm{Zn}$ e $\mathrm{MnO}_{2}$ é, respectivamente, 2 e 1 . Então, supondo-se que a transferência de carga seja $100 \%$ na inserção do próton ou oxidação do $\mathrm{Zn}$, o valor da capacidade específica para o eletrodo de $\mathrm{Zn}$, utilizando-se da expressão da Lei de Faraday é $\mathrm{m}_{\mathrm{Zn}}$ x 0,8198 $\mathrm{Ah} \mathrm{g}^{-1}$ e para $\mathrm{MnO}_{2}$ é $\mathrm{m}_{\mathrm{MnO} 2}$ x 0,3083 $\mathrm{Ah} \mathrm{g}^{-1}$, onde $\mathrm{m}$ é a massa, em g, de $\mathrm{Zn}$ ou de $\mathrm{MnO}_{2}$. A capacidade específica para o cátodo com $10,42 \%$ de grafite é $0,206 \mathrm{Ah} \mathrm{g}^{-1}, 0,219 \mathrm{Ah} \mathrm{g}^{-1}$ quando o cátodo é composto por $5 \%$ de grafite e para o $\mathrm{Zn}$, é de $0,614 \mathrm{Ah} \mathrm{g}^{-1}$. Portanto, essas pilhas possuem capacidade limitada pelo cátodo, ou seja, o $\mathrm{MnO}_{2}$. Isto implica que, se as pilhas estudadas no presente trabalho forem descarregadas muito além de $0,8 \mathrm{~V}$, poderá ser gerado $\mathrm{H}_{2}$. $\mathrm{O}$ consumo total do $\mathrm{MnO}_{2}$ no eletrodo pode ativar a reação $\mathrm{Zn}+2 \mathrm{H}_{2} \mathrm{O} \rightarrow \mathrm{H}_{2}+\mathrm{Zn}(\mathrm{OH})_{2}$, uma vez que o potencial do $\mathrm{Zn}$ em relação à grafite do cátodo é suficiente para dar início a esta. As Figuras 3 e 4 mostram as curvas de descarga de uma pilha comercial AA utilizando condições galvanostáticas e através do acoplamento com uma resistência.

Ambas as condições permitem a determinação dos parâmetros energéticos, como por ex., a capacidade específica. No caso galvanostático, como já mencionado, é suficiente multiplicar a corrente na cela pelo tempo de descarga. Já para a resistência

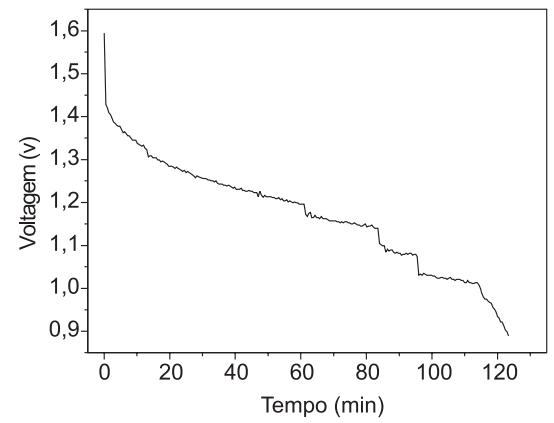

Figura 3. Exemplo de uma curva de descarga de uma pilha comercial AA utilizando uma resistência de carga de 11,4 ohms

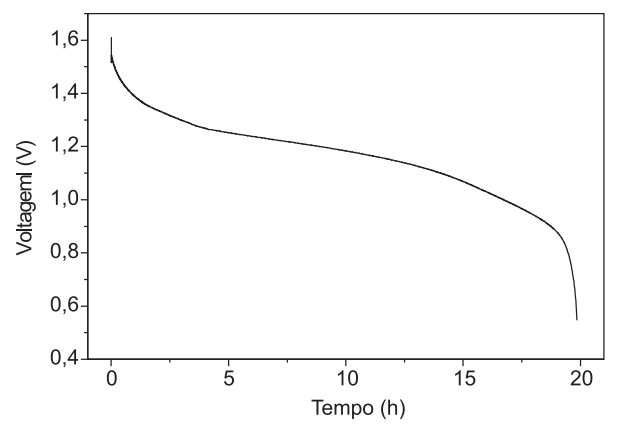

Figura 4. Exemplo de uma curva de descarga de uma pilha comercial, utilizando condições galvanostáticas (corrente constante igual a $10 \mathrm{~mA}$ ) 
acoplada, a capacidade específica é calculada dividindo-se a voltagem da pilha pela resistência a cada instante e integrando a curva obtida no intervalo de 1,6 a 0,9 V. Entretanto, a principal diferença entre os dois modos de descarga é o efeito da resistência interna sobre a voltagem da pilha. As curvas de descarga em ambos os modos contêm explicitamente a variação de resistência do eletrodo ou a queda ôhmica iR ( $\mathrm{i}=$ corrente do galvanostato, $\mathrm{R}$ resistência da pilha ou eletrodo) que é observada no início da polarização da pilha. No caso galvanostático, a influência da resistência sobre a variação de voltagem pode ser determinada porque a corrente é constante, enquanto que no caso da descarga com resistência acoplada, esta influência é mais difícil de ser observada porque i e $\mathrm{R}$ podem variar ao mesmo tempo. Efeitos resistivos no eletrodo são responsáveis pelo aquecimento da bateria, como já mencionado (efeito Joule). Este aquecimento, que é a perda de energia que não pode ser convertida em trabalho, pode levar à degradação dos materiais que compõem o eletrodo. Em alguns casos ele pode provocar o aumento da pressão de vapor do eletrólito com consequiente vazamento ou explosão da bateria.

\section{INFLUENCIA DA MORFOLOGIA E DA CONCENTRAÇÃO DE GRAFITE NAS CURVAS DE DESCARGAS DE PILHAS ALCALINAS}

Do ponto de vista prático, a principal forma de se estudar as propriedades físico-químicas de uma pilha é através de curvas de descarga em condições de corrente constante (condições galvanostáticas). Este tipo de caracterização é extremamente im-

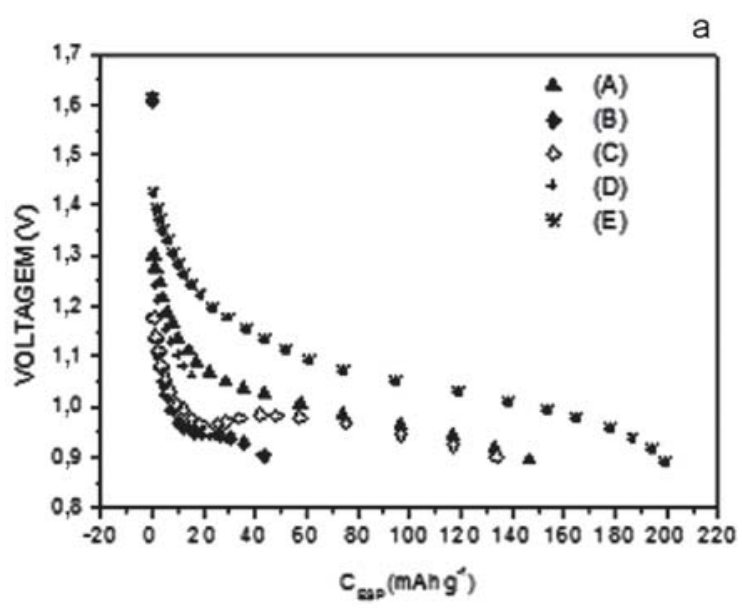

b

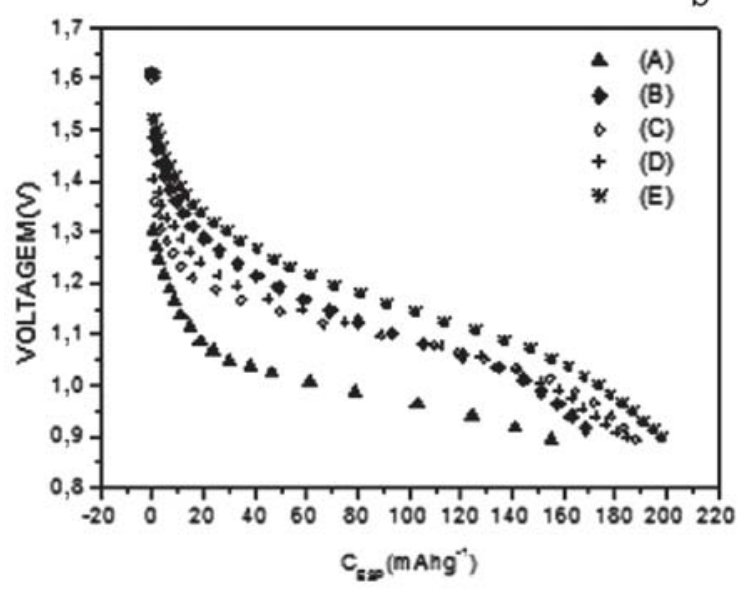

Figura 5. Curvas de descarga de pilhas alcalinas $\mathrm{MnO}_{2}$ IZn preparadas com grafite de diferentes parâmetros e em diferentes concentrações portante para o entendimento da influência dos materiais na resposta elétrica da pilha.

A Figura 5 mostra as curvas de descarga das pilhas alcalinas em função das grafites mostradas na Tabela 1 e da quantidade de grafite usada no cátodo, 5\% (Figura 5a) e 10,42\% (Figura 5b). Todas as pilhas apresentam uma resposta de voltagem em função da capacidade específica nominal, que apresenta uma grande variação no início da polarização. Esta variação é a queda ôhmica e a Figura 5 mostra que esta é menor quanto maior a quantidade de grafite usada nos eletrodos (Figura $5 b$ ).

$\mathrm{O}$ aumento da concentração de grafite no cátodo implica em um valor menor de $R$, que é a soma das resistências no cátodo, eletrólito e ânodo. Nota-se também que dependendo do tipo e da quantidade de grafite usada, a variação de potencial apresenta um andamento diferenciado.

As variações nos perfis de voltagem das pilhas $\mathrm{MnO}_{2} / \mathrm{Zn}$ dependem da diferença de potencial do ânodo e do cátodo. O potencial no ânodo de Zn segue a Equação de Nernst, ou seja, é função da concentração de $\mathrm{Zn}$ e $\mathrm{ZnO}$. Já o potencial do $\mathrm{MnO}_{2}$ ou de materiais como aqueles usados em baterias de íons de $\mathrm{Li}^{16,17}$ apresentam uma variação de potencial que depende do potencial químico de íons e elétrons ${ }^{18,19}$. Para os eletrodos preparados com $5 \%$ de grafite (Figura 5a), a variação da voltagem com a capacidade e a queda ôhmica é menor para as grafites E e A, enquanto que para as demais grafites esta variação se apresenta muito maior, a ponto de provocar uma grande redução na capacidade da pilha (grafite D) e uma variação grande e irregular no caso das grafites $\mathrm{C}$ e B. Esta influência, devido aos diferentes parâmetros da grafite utilizada, torna-se menor quando a concentração da mesma no eletrodo é aumentada (Figura 5b). Para as pilhas preparadas com $10,42 \%$ de grafite, a maior variação de potencial e queda ôhmica ocorreu para a grafite A. Na Figura 5a nota-se ainda que, para a grafite $\mathrm{C}$, a diferença de potencial da pilha apresenta, depois da queda inicial, um pequeno aumento seguido de um declínio. Este aumento pode estar associado com um atraso na voltagem do ânodo e/ou decréscimo na resistência do cátodo e/ou eletrólito. Finalmente, os resultados mostram que a capacidade específica de todas as pilhas descarregadas até $0,9 \mathrm{~V}$ foi sempre menor que o valor teórico de 0,206 $\mathrm{Ah} \mathrm{g}^{-1}$ (Figura 5a), 0,219 $\mathrm{Ah} \mathrm{g}^{-1}$ (Figura $5 b)$. A diferença entre as capacidades foi sempre menor nas pilhas preparadas com maior quantidade de grafite. Também se verificou que as pilhas que apresentaram melhor capacidade, independente da concentração de grafite, foram aquelas preparadas com a grafite E.

Existem várias razões que podem explicar a não obtenção da capacidade específica teórica das pilhas alcalinas como as estudadas no presente trabalho. A capacidade específica experimental de uma pilha depende da massa ativa de seu eletrodo que está em contato com o eletrólito. Assim, se parte da massa de $\mathrm{MnO}_{2}$ utilizada na preparação do eletrodo não estiver em contato com o eletrólito, esta não participará da reação e, portanto, a capacidade teórica obtida é sobre-valorizada. Outro fator que pode contribuir para uma redução da capacidade é o aumento da resistência da bateria durante a descarga. No caso da pilha alcalina, este aumento é associado de forma dominante pelo cátodo $\mathrm{MnO}_{2}$, uma vez que o $\mathrm{Zn}$ é um metal e o $\mathrm{MnO}_{2}$ é um óxido isolante. Um aumento na resistência do cátodo implica em uma redução da cinética da reação de inserção de prótons no $\mathrm{MnO}_{2}$. A inserção de prótons torna-se mais difícil porque os elétrons injetados no cátodo não são suficientes para suprir o gradiente de concentração iônica e eletrônica existente no interior dos grãos. Tal deficiência provoca o surgimento de reações paralelas como a geração de $\mathrm{H}_{2}$ que pode introduzir bolhas no separador, o que dificulta o contato entre as interfaces. Isto explica porque o aumento da resistência irá reduzir o tempo de descarga da pilha e, conseqüentemente, sua capacidade de descarga. 
Assim, verifica-se das curvas de descarga galvanostática que a quantidade e a variação dos parâmetros da grafite usada na preparação do eletrodo de dióxido de manganês são determinantes no desempenho das pilhas alcalinas.

\section{ROTEIROS DE PREPARAÇÃO E CARACTERIZAÇÃO FÍSICO-QUÍMICA DE UMA PILHA ALCALINA}

Todos os conceitos discutidos anteriormente podem ser aplicados em aula experimental através da construção de uma pilha e da caracterização da mesma ou de uma pilha comercial. A seguir, apresentamos o roteiro que foi aplicado em laboratório como experimento da disciplina de físico-quimica experimental, com duração de 6 h-aula.

\section{Construção da cela eletroquímica}

A Figura 6 mostra o esquema da pilha alcalina que foi construída no experimento realizado pelos alunos. Os coletores de corrente usados foram de $\mathrm{Ni}$ ou aço revestido com Ni. Estes possuem uma rosca, que tem a finalidade de permitir o ajuste da espessura da cavidade onde é pastilhada a mistura catódica. Um anel de vedação é usado para evitar evaporação do solvente do eletrólito.

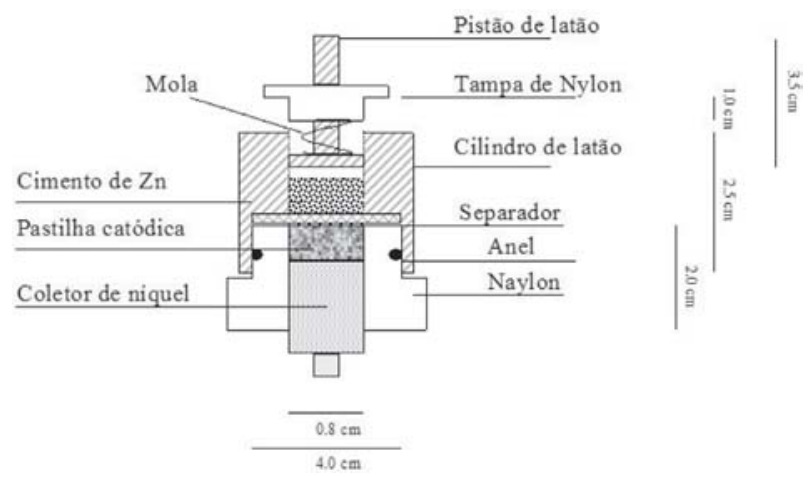

Figura 6. Ilustração da cela eletroquímica utilizada no estudo da pilha $\mathrm{MnO}_{2} / \mathrm{Zn}$ em função do conteúdo de grafite no pólo positivo. Na figura são indicadas as principais dimensões da cela e os valores para quantidade de massa utilizada no ânodo e no cátodo

Este tipo de ajuste é necessário para posicionar a superfície da pastilha catódica na posição onde vem inserido o separador. Um corpo cilíndrico de latão é fixo à base catódica por um anel tipo "o'ring". Este recipiente funciona como reservatório para o cimento anódico. O fechamento da cela é realizado através de uma tampa de Nylon ou latão, que possui rosca revestida com fita de teflon. Esta tampa comprime uma mola que pressiona um pistão de latão sobre o cimento de $\mathrm{Zn}$. A cela assim obtida pode operar na vertical ou horizontal, com o auxílio de uma garra.

\section{Roteiro experimental da montagem da pilha}

A tarefa de preparação do cátodo é, de fato, o processo mais delicado de preparação das pilhas alcalinas atuais. O papel do carbono condutor é extremamente decisivo neste processo, porque define a resistência do cátodo da pilha alcalina, como mostra o presente experimento. No caso das pilhas alcalinas é sempre importante utilizar um carbono condutor com superfície hidrofílica pois caso contrário, torna-se difícil o acesso do eletrólito no interior do cátodo ou sua percolação. Entretanto, a concentração de carbono é extremamente importante neste processo. $\mathrm{O}$ excesso de car- bono no cátodo pode comprometer o contato da massa ativa de $\mathrm{MnO}_{2}$ com o eletrólito e gerar reações paralelas que ocasionam a formação de $\mathrm{H}_{2}$. A presença deste gás aumenta a pressão interna da cela, formando bolhas entre nas várias interfaces no interior da cela, o que pode provocar um grande aumento da resistência da pilha. Assim, o ideal na preparação de uma pilha alcalina é utilizar a menor quantidade possível de grafite no cátodo. Entretanto, os resultados apresentados demonstram que a quantidade de grafite que pode ser utilizada em uma pilha depende muito também das propriedades dessa grafite. A seguir, é apresentado o roteiro a ser seguido pelo estudante:

A) misturar de forma homogênea $\mathrm{MnO}_{2}$, grafite e $\mathrm{CaCO}_{3}$ em um almofariz. Adicionar à mistura cerca de $0,121 \mathrm{~g} / 0,24 \mathrm{~mL}(16 \%$ do peso total do cátodo) de $\mathrm{KOH} \mathrm{9M}$;

B) pressionar a mistura a $0,15 \mathrm{t}$ usando o pastilhador de PVC por 3 min. Retirar a pressão e a pastilha, esperar 5 min. Não esquecer de desrosquear o eletrodo até o fim para não estragar a rosca;

C) ao retirar a pastilha, pulverizá-la novamente no almofariz. Repetir 3 vezes;

D) posicionar a superfície da pastilha exatamente no plano da cela, onde serão posicionados os separadores;

E) (opcional) conectar bomba de vácuo na parte inferior da cela e manter a bomba ligada por alguns segundos, o suficiente para gotejar a solução (a função da bomba é facilitar a penetração do eletrólito);

F) repousar por $30 \mathrm{~min}$ os separadores em $2 \mathrm{~mL}$ de $\mathrm{KOH} \mathrm{9,0}$ M; inserir os dois separadores (área de $4 \mathrm{~cm}^{2}$ ) sobre o eletrodo;

G) fechar a cela com a parte superior pressionando até o encaixe perfeito da mesma (o anel de o'ring deve ser posicionado);

$\mathrm{H})$ adicionar a pasta de $\mathrm{Zn} /$ carboxi-metil celulose/KOH preparada como descrito a seguir: H.1. misturar de forma homogênea $\mathrm{Zn}$ e carboxi-metil celulose em um almofariz e adicionar $0,25 \mathrm{~mL}$ de $\mathrm{KOH}$ 9,0 M; H.2. inserir o pistão de contato no ânodo da cela (parte com $\mathrm{Zn})+$ mola e fechar o suficiente para que haja o contato elétrico;

I) conectar ao equipamento em circuito aberto (o potencial deverá ser $~ 1.6 \mathrm{~V}$.

Dar início aos testes eletroquímicos de preferência após 30 min de conexão, tempo suficiente para checar se o potencial da cela de circuito aberto se encontra estável.

A seguir são apresentados, na Tabela 2, os principais dados para a montagem da pilha descrita no roteiro acima.

Tabela 2. Resumo dos principais parâmetros experimentais necessários para a montagem de um protótipo de pilha alcalina e uma sugestão de corrente de descarga para teste

\begin{tabular}{|c|c|}
\hline Densidade de Corrente $\left(\mathrm{mA} \mathrm{g}^{-1}\right)$ & 13,23 \\
\hline $\begin{array}{l}\text { Capacidade para cut-off de } \\
0,9 \mathrm{~V} \text { ou } 0,9 \mathrm{e} / \mathrm{MnO}\end{array}$ & $277,47 \mathrm{mAh} / \mathrm{g}$ \\
\hline $\begin{array}{l}0,9 \mathrm{~V} \text { ou } 0,9 \mathrm{e} / \mathrm{MnO}_{2} \\
\text { Corrente }(\mathrm{mA})\end{array}$ & $10 \mathrm{~mA}$ \\
\hline Massa ou $\% \mathrm{MnO}_{2}$ & $88,54 \%$ ou $0,669 \mathrm{~g}$ \\
\hline $\begin{array}{l}\text { Massa ou } \% \text { Grafite } \\
\text { Volume de } \mathrm{KOH} 9,0 \mathrm{M} \text { usado no } \\
\text { cátodo }\left(\mathrm{MnO}_{2} / \text { grafite/cal }\right)\end{array}$ & $\begin{array}{c}10,42 \% \text { ou } 0,0778 \mathrm{~g} \\
0,24 \mathrm{~mL}\end{array}$ \\
\hline $\begin{array}{l}\text { Massa total do cátodo } \\
\left(\mathrm{MnO}_{2} / \text { grafite/cal }\right)\end{array}$ & $0,756 \mathrm{~g}$ \\
\hline $\begin{array}{l}\text { Massa total do ânodo } \\
\text { (Zn/carboxi-metil celulose) }\end{array}$ & $0,787 \mathrm{~g}$ de $\mathrm{Zn}$ e $0,0077 \mathrm{~g}$ de $\mathrm{CMC}$ \\
\hline $\begin{array}{l}\text { Volume de } \mathrm{KOH} 9,0 \mathrm{M} \\
\text { usado no ânodo de } \mathrm{Zn}\end{array}$ & $0,25 \mathrm{~mL}$ \\
\hline
\end{tabular}




\section{Caracterização das pilhas e questões}

Para orientar os alunos na realização do experimento durante a aula e na elaboração do relatório, foi preparado o roteiro abaixo. Este contém a metodologia efetivamente aplicada, ou seja, os alunos iniciavam a montagem das pilhas e depois, durante a descarga das mesmas, o docente aproveitava para esclarecer, questionar e discutir os conceitos que deveriam posteriormente ser apresentados, como forma de avaliação, no relatório da atividade.

i) Montar a pilha seguindo roteiro descrito anteriormente e deixar cerca de 30 min em repouso para a percolação do eletrólito por todo o eletrodo de $\mathrm{MnO}_{2}$;

ii) descarregar uma pilha comercial modelo AA em condições galvanostáticas até $0,9 \mathrm{~V}$, ou seja, um equipamento que mantém a corrente de descarga constante enquanto o potencial da pilha varia;

iii) estimar a massa de material ativo $\left(\mathrm{MnO}_{2}\right)$, observar a queda ôhmica e calcular a resistência da pilha comercial;

iv) montar um circuito, utilizando uma pilha e uma associação de resistores (resistência equivalente de 500 ohms ou menor, por ex.). Utilizar um multímetro para medida da voltagem vs tempo e da corrente de descarga vs tempo. A partir desta curva, de corrente vs tempo, determine a capacidade de carga;

v) discuta a influência da quantidade de grafite na resistência da pilha;

vi) qual a diferença entre descarregar uma pilha com corrente constante (condições galvanostáticas) e utilizando uma associação de resistores?

vii) qual a diferença entre cátodo e ânodo na eletroquímica e na parte elétrica?

viii) escreva as reações nos eletrodos da pilha montada.

ix) quais são os fatores que provocam curto-circuito na cela?

$\mathrm{x})$ calcule a capacidade específica para as curvas de descarga com corrente constante e com associação de resistores.

xi) estime a quantidade de $\mathrm{MnO}_{2}$ presente em uma pilha comercial.

xii) do ponto de conversão de energia química em elétrica, qual seria a melhor forma de descarregar uma bateria, usar uma resistência de carga ou um galvanostato?

\section{CONCLUSÕES}

Neste trabalho, mostramos como a preparação e a descarga de pilhas alcalinas tornam-se um instrumento útil para a abordagem de uma série de conceitos de interesse no aprendizado de reações de oxidação e redução associadas com o armazenamento de energia, com a termodinâmica e com o entendimento operacional de uma pilha alcalina. Esta pilha permite abordar um tipo de reação "redox" que envolve interface (Zn) e reações de inserção/extração de íons no $\mathrm{MnO}_{2}$. A preparação do experimento é relativamente simples e os materiais utilizados são de baixo custo e não tóxicos, facilitando sua implementação em um laboratório de ensino.

Estes materiais foram obtidos gratuitamente nas empresas Nacional de Grafite (grafite) e Eletromanganês $\left(\mathrm{MnO}_{2}\right.$ eletrolítico). A montagem e caracterização das pilhas podem ser conduzidas durante 4 a 6 h de laboratório, dependendo da capacidade específica ou da massa de material ativo utilizada no experimento. A prática apresentada permite um bom contato com questões que podem estar envolvidas na produção industrial de pilhas, um tópico de interesse para cursos de Química Tecnológica.

É importante mencionar que, quando o laboratório não possuir equipamentos para o registro contínuo do potencial do eletrodo em relação ao tempo durante a polarização da pilha, a aquisição do tempo pode ser realizada com o auxílio de um cronômetro. Outra opção é a realização de pulsos de corrente, seguido de um relaxamento. Mede-se o tempo de duração do pulso (carga descarregada) e associa-se a este valor o potencial tomado no final do relaxamento (circuito aberto), quando o mesmo tende a se estabilizar. O tempo de relaxamento dependerá da intensidade e duração do pulso de corrente aplicado na descarga da pilha, bem como da capacidade nominal da pilha. Estas observações são importantes, pois a medida da voltagem de pilhas ou baterias pode acarretar em descargas, quando a resistência interna do voltímetro não é suficientemente elevada $\left(<10^{8} \mathrm{ohms}\right)$. Neste caso, recomenda-se que a medida da diferença de potencial da pilha seja realizada rapidamente, o suficiente para não provocar grandes oscilações na medida durante a leitura. Por fim, deve ser mencionado ainda que em circuitos elétricos o fluxo de corrente segue o sinal do campo elétrico, enquanto na eletroquímica o fluxo de elétrons é o da corrente. Assim, na eletroquímica, o cátodo é efetivamente o pólo negativo, mas em circuitos elétricos (baterias e pilhas comerciais) convenciona-se o mesmo como pólo positivo e o ânodo como negativo.

Dos 12 grupos de alunos submetidos à atividade, notou-se que a maior dificuldade estava na determinação dos pólos das pilhas e conexões elétricas com a resistência, o galvanostato e o multímetro. A determinação do tempo durante a descarga usando a resistência também foi um ponto de dificuldade. No início da descarga da pilha ocorre uma grande variação de corrente e muitos alunos não tomam o cuidado de realizar várias leituras neste período.

\section{AGRADECIMENTOS}

À FAPESP, à empresa Nacional de Grafite e aos alunos de Físico-química experimental da $38^{a}$ turma de Química da FFCLRP USP. J. M. Rosolen agradece ainda ao CNPq e à CAPES.

\section{REFERÊNCIAS}

1. Atkins, P. W.; Physical-Chemistry, Editora Oxford: Oxford, 1990, p. 243.

2. McQuarrie, D. A.; Simon, J. D.; Physical Chemistry: A Molecular Approach, University Science Books: Sausalito, 1997.

3. Moore, W. J.; Físico-Química, Ed. Edgar Blucher e EDUSP: São Paulo, 1976

4. Linden, D.; Handbook of Batteries and Fuel Cells, $2^{\text {nd }}$ ed., McGraw-Hill: New York, 1995.

5. Vincent, C. A.; Modern Batteries, $2^{\text {nd }}$ ed., Edward Arnold: Maidenhead, 1998.

6. Sharma, Y.; Aziz, M.; Yusof, J.; Kordesch, K.; J. Power Sources 2001, 94, 129.

7. Patrice, R.; Gerand, B.; Leriche, J. B.; Seguin, L.; Wang, E.; Moses, R.; Brandt, K.; Tarascon, J. M.; J. Electrochem. Soc. 2001, 148, A448.

8. Rosolen, J. M.; Decker, F.; Gorenstei, A.; Torresi, R. M.; Torresi, S. I. C J. Electroanal. Chem. 1993, 354, 273.

9. Megahed, S.; Scrosati, B.; J. Power Sources 1994, 51, 79.

10. David, W. I. F.; Thackeray, M. M.; Bruce, P. G.; Goodenough, J. B.; Mater. Res. Bull. 1984, 19, 99.

11. Wells, A. F.; Structural Inorganic Chemistry, Editora Oxford University: London, 1962, p. 462.

12. Laurindo, E. A.; Amaral, F. A.; Santos, M. L.; Ferracin, L. C.; Carubelli, A.; Bocchi, N.; Rocha-Filho, R. C.; Ouim. Nova 1999, 22, 600.

13. Cullity, B. D.; Elements of $X$-ray diffraction, Addison-Esley Publishing Company Inc.: Massachusets, USA, 1967.

14. Brunauer; S.; Emmett; P.; Teller, E.; J. Am. Chem. Soc. 1938, 60, 309

15. Lippi, R.; Suzuki, N. M.; Rosolen, J. M.; Technical News from National Instruments 2000, 1, 3 .

16. Passerini, S.; Smyrl, W. H.; Berrettoni, M.; Tossici, R.; Rosolen, J. M.; Marassi, R.; Decker, F.; Solid State Ionics 1996, 47, 5.

17. Passerini, S. Rosolen, J. M.; Scrosati, B ; J Power Sources 1993, 45, 333

18. Gerischer, H.; Decker, F.; Scrosati, B.; J. Electrochem. Soc. 1993, 140, 315.

19. Reimers; J. N.; Dahn, J. R.; Phys. Rev. B: Condens. Matter Mater. Phys. 1993, 47, 2995. 